\title{
Atividades físicas de lazer por universitários: inquéritos repetidos nos anos de 2010, 2012 e 2014
}

\section{Leisure-time physical activities practice by undergraduate: repeated surveys in years} of 2010,2012 e 2014

\section{AUTORES \\ Thiago Ferreira de Sousa ${ }^{1,2}$ (D) \\ Dayana Chaves Franco ${ }^{3}$ (D) \\ Gildeene Silva Farias ${ }^{1}$ \\ Gerleison Ribeiro Barros ${ }^{1}$ (DD \\ 1 Programa de Pós-Graduação em Educação Física. Universidade Federal do Triângulo Mineiro, Uberaba, Minas Gerais, Brasil \\ 2 Centro de Formação de Professores. Universidade Federal do Recôncavo da Bahia, Amargosa, Bahia, Brasil. \\ 3 Centro Universitário de Maringá, Maringá, Paraná, Brasil.}

\section{CONTATO}

Thiago Ferreira de Sousa

tfsousa_thiago@yahoo.com.br

Universidade Federal do Recôncavo da Bahia. Centro de Formação de Professores. Avenida Nestor de Mello Pita, no 535 (caixa postal: 64), Centro, Amargosa, Bahia, Brasil. CEP: 45300-000.

DOI

$10.12820 /$ rbafs.24e0099

\begin{abstract}
RESUMO
O objetivo deste estudo foi comparar as proporções de prática de diferentes tipos de atividades físicas no lazer por universitários entre os anos de 2010, 2012 e 2014. Realizou-se três inquéritos repetidos com amostras de universitários de uma instituição de ensino superior do estado da Bahia. As modalidades foram mensuradas por meio de um questionário validado para aplicação em universitários, tendo sido os seguintes tipos: ciclismo, voleibol, natação, caminhada em esteira, hidroginástica, surfe, caminhada em locais abertos, futebol, basquetebol, handebol, corrida em locais abertos, ginástica aeróbica, lutas, tênis, musculação, corrida em esteira e ginástica em geral com uso de pesos e trampolins. As comparações foram realizadas utilizando o teste qui-quadrado para tendência linear. $\mathrm{O}$ nível de significância foi de 5\%. Participaram do estudo 1.084, 1.085 e 1.041 universitários, nos anos de 2010, 2012 e 2014, respectivamente. A modalidade mais praticada nos três inquéritos foi a musculação, seguida do futebol. A prática das modalidades de corrida $(2010=6,9 \% ; 2012=8,8 \% ; 2014=11,0 \%)$ e de lutas $(2010=4,2 \% ; 2012=4,5 \% ; 2014=7,6 \%)$ aumentaram ao longo dos inquéritos. Houve a diminuição na prática da ginástica com uso de pesos/trampolins ( $2010=3,7 \% ; 2012=1,9 \% ; 2014$ $=1,4 \% ; p=0,001)$. Conclui-se que a tendência de atividade física de lazer ao longo do tempo em universitários foi diferente entre as modalidades e em relação as características sociodemográficas e de vínculo com a universidade, com aumento de praticantes de lutas e corridas, e diminuição dos adeptos da ginástica com pesos/trampolins.
\end{abstract}

Palavras-chave: Atividade motora; Exercício; Estudantes; Estudos transversais.

\section{ABSTRACT}

The aim of this study was to compare the proportions of practices of different types of leisure-time physical activities by college students in 2010, 2012 and 2014. Three repeated surveys conducted with college from a higher education institution from state Babia, Brazil. The modalities were measured through a questionnaire validated for application in college, having been the following types: cycling, volleyball, swimming, treadmill walking, water aerobics, surfing, walking in local places, soccer, basketball, handball, outdoor running, aerobics gymnastics, fights or martial arts, tennis, weight training, treadmill running and general gymnastics using weights and trampolines. As comparisons performed via chi-square test for linear trend. The level of significance was 5\%. Participated 1,084, 1,085 and 1,041 college students in 2010, 2012 and 2014, respectively. The most practiced modality in the three surveys was about weight training, followed by soccer. The practice of outdoor running $(2010=6.9 \% ; 2012=8.8 \% ; 2014=11.0 \%)$ and martial arts $(2010$ $=4.2 \% ; 2012=4.5 \% ; 2014=7.6 \%)$ increased throughout the investigations. There was a decrease in the practice of gymnastics $(2010=3.7 \% ; 2012=1.9 \% ; 2014=1.4 \% ; p=0.001)$. It can be concluded that the trend of leisure-time physical activities over time among college students was different according with the type of modalities, with an increase in practitioners of fights or martial arts and running, and decrease in the number of gymnastics practitioners using weights and trampolines.

Keywords: Motor activity; Exercise; Students; Cross-sectional studies.

\section{Introdução}

Os baixos níveis de atividade física representam um problema de saúde pública no mundo e um dos fatores de risco para a mortalidade por todas as causas ${ }^{1}$. A prática regular de atividade física contribui com potenciais benefícios à saúde como fator de proteção e prevenção das doenças crônicas não transmissíveis ${ }^{1}$, no entanto, nota-se que a adoção desse comportamento por universitários não tem ocorrido de forma frequente ${ }^{2,3}$.

Em estudo de revisão sistemática, foi mostrado que as prevalências de inatividade física ou baixos níveis de atividades físicas em universitários brasileiros variam de zero a $78,9 \%^{2}$, e tais valores dependem do perfil de vínculo com a Universidade, haja vista que estudantes 
do curso de Educação Física tendem a ser mais ativos no lazer ${ }^{3}$. Em pesquisa com universitários de 23 países, observaram-se elevadas prevalências de inatividade física no lazer, ou seja, não ter praticado nenhuma atividade física no lazer nas últimas duas semanas, tendo sido 23\% para aqueles da Europa Ocidental e Norte dos Estados Unidos, 30\% da Europa Central e Oriental, 39\% referente aos estudantes do Mediterrâneo, 42\% para o Pacífico Asiático e 44\% para os países em desenvolvimento ${ }^{4}$.

Sobre as preferências de prática de atividades físicas de lazer pelo público universitário, pode-se citar futebol, corrida e musculação ${ }^{5}$. No entanto, nota-se a lacuna quanto a caracterização de tais informações ao longo do tempo, de modo a demonstrar a estabilidade ou não da prática de diferentes tipos de atividades físicas durante o tempo livre de universitários, conforme já observado em outros estudos com adultos e idosos, brasileiros, que mostraram o aumento da proporção de praticantes entre 2006 e $2016^{6}$ e noruegueses, que observaram o declínio da prática entre 1979-80 e $2001^{7}$.

Soma-se a isso, a relevância em destacar o comportamento de adesão ao longo do tempo nas diversas modalidades, em função das características que podem melhor discriminar o perfil de prática, pois homens ${ }^{3,5}$, universitários mais jovens ${ }^{5}$, sem companheiro ${ }^{5}$, do período de estudo diurno ${ }^{3}$ e de cursos da área das Ciências da Saúde ${ }^{3,5}$ estão mais envolvidos com a prática de atividades físicas de lazer de modo geral ${ }^{3}$, ou específicas, como musculação, futebol e corrida ${ }^{5}$ Importante ressaltar que o público universitário cresceu nos últimos anos no Brasil ${ }^{8}$, e o ingresso na vida acadêmica, como etapa de transição entre o final da adolescência e início da idade adulta ${ }^{9}$, com o aumento de atividades e ocupações acadêmicas ${ }^{10,11}$, pode favorecer o surgimento de condutas negativas a saúde, como o menor envolvimento em atividades físicas ${ }^{12}$.

A apresentação de informações relacionadas ao perfil de prática de diferentes modalidades de atividades físicas de lazer, em universitários, pode permitir que as Universidades destinem esforços direcionados à manutenção desse comportamento durante a vida universitária. Além disso, torna-se essencial entender esse comportamento, pois os universitários compõem a população em geral, o que reforça a necessidade de ações por meio de políticas públicas que possam favorecer a adoção de um estilo de vida ativo ao longo da vida. Deste modo o objetivo deste estudo foi comparar as proporções de prática de diferentes tipos de atividades físicas no lazer por universitários entre os anos de 2010, 2012 e 2014.

\section{Método}

Esta pesquisa faz parte do estudo MONISA (Monitoramento dos Indicadores de Saúde e Qualidade de Vida em Acadêmicos), realizado em uma universidade pública do estado da Bahia. Os dados deste estudo fazem parte dos inquéritos realizados nos anos de 2010, 2012 e 2014. Os detalhamentos metodológicos foram apresentados em estudo anterior ${ }^{13}$. O projeto MONISA foi aprovado pelo comitê de ética em pesquisa local, sob o número 382/2010 e os participantes assinaram termo de consentimento livre e esclarecido antes da participação.

A população deste estudo, em cada ano de inquérito, foi composta pelos universitários matriculados na universidade, no segundo semestre letivo, nos cursos de graduação presenciais. Foram excluídos os ingressantes no segundo semestre letivo (calouros). As amostras foram calculadas tendo como base a população alvo de cada ano de inquérito $(2010=5.461 ; 2012=5.767$; 2014 = 5.224), nível de confiança de 95,0\%, prevalência estimada em $50,0 \%$, erro aceitável de três pontos percentuais, acréscimo de 20,0\% para perdas/recusas e mais $15,0 \%$ para o controle das variáveis de confusão nas análises ajustadas. Com base nesses parâmetros, as amostras calculadas em cada ano de inquérito foram: 1.232 em 2010, 1.243 em 2012 e 1.223 em 2014.

Posteriormente, a amostra calculada foi estratificada considerando a proporção dos universitários entre os cursos de graduação pertencentes em cada ano (30 cursos em 2010; 34 cursos em 2012; e 33 cursos em 2014), período de estudo diurno (matutino e vespertino) e noturno, e ano de ingresso na universidade, classificados em quatro categorias, pois a maioria dos cursos apresentava a quantidade mínima de quatro anos para a conclusão de forma regular, sendo no inquérito de 2010 estabelecido como estratos os anos de entrada de 2010, 2009, 2008, e 2007 e anteriores; no inquérito de 2012 os seguintes estratos, os anos de entrada de 2012, 2011,2010, e 2009 e anteriores; e no inquérito de 2014 os estratos foram os anos de 2014, 2013, 2012, e 2011 e anteriores.

A unidade amostral foram os universitários, sorteados por procedimento de seleção aleatória simples, em cada estrato, utilizando-se, para tanto, a lista de matrícula em ordem alfabética, e os sorteios realizados por meio do programa Research Randomizer. Os universitários selecionados foram procurados na instituição, nas salas de aula dos seus cursos, em dias e horários diferentes, em até três tentativas. Aqueles que informaram não 
ter interesse em participar foram considerados como recusa. Para as perdas e recusas não houve reposições.

Nos três inquéritos, as coletas de dados foram realizadas de setembro a novembro, nas salas da universidade, de forma individualizada ou em grupos de até 40 universitários, conforme conveniência dos voluntários e dos professores que disponibilizaram parte do tempo da aula para a realização da coleta de dados. Em todas as situações, a aplicação ocorreu resguardando as mesmas condições, como a sala isolada para a aplicação, com a presença de um aplicador para sanar eventuais dúvidas e previamente a participação foi informado o objetivo da pesquisa e a forma de participação (autopreenchimento do instrumento). O tempo médio de resposta ao instrumento foi de 30 minutos. $O$ treinamento da equipe de coleta de dados (professores e universitários da instituição, não pertencentes as amostras) ocorreu nos meses de julho a agosto de cada ano dos inquéritos. As informações foram obtidas via questionário ISAQ-A (Indicadores de Saúde e Qualidade de Vida de Acadêmicos) ${ }^{14}$.

A prática de atividades físicas de lazer em uma semana habitual foi mensurada por meio de uma pergunta com as seguintes opções de modalidades: ciclismo, voleibol, natação, caminhada em esteira, hidroginástica, surfe, caminhada em locais abertos, futebol, basquetebol, handebol, corrida em locais abertos, ginástica aeróbica, lutas ou artes marciais, tênis, musculação, corrida em esteira e ginástica em geral com uso de pesos e trampolins. Foi considerado como desfecho a informação positiva de prática, independente da frequência de dias e quantidade de horas e minutos da prática, sendo possível informar mais de uma modalidade. Durante a aplicação do instrumento foi orientado que as práticas que não tinham opção de modalidade, que fossem respondidas conforme ao tipo de atividade que melhor representasse a prática. Além disso, foi possível informar outras modalidades em três opções de resposta abertas, sendo essas modalidades quando possível reclassificadas dentre as opções listadas, como por exemplo, o agrupamento das diferentes manifestações de lutas ou artes marciais em uma única opção. Decidiu-se não incluir neste estudo as informações das respostas as perguntas abertas, pois apresentavam menores prevalências e não apresentam informações de comparação ao longo dos inquéritos. Os níveis de reprodutibilidade dos tipos de atividades físicas de lazer variaram de Kappa de 0,35 para ciclismo a 1,00 para futebol ${ }^{14}$.

A variável independente foram os anos dos inquéritos, correspondentes aos anos de 2010, 2012 e 2014.
As variáveis para estratificação nas análises foram sexo (masculino e feminino), faixa etária em tercis (Inquérito de $2010=1^{\circ}$ tercil, 17 a 20 anos; $2^{\circ}$ tercil, 21 a 23 anos; $3^{\circ}$ tercil, 24 a 52 anos; Inquérito de $2012=1^{\circ}$ tercil, 17 a 20 anos; $2^{\circ}$ tercil, 21 a 23 anos; $3^{\circ}$ tercil, 24 a 54 anos; Inquérito de $2014=1^{\circ}$ tercil, 17 a 20 anos; $2^{\circ}$ tercil, 21 a 23 anos; $3^{\circ}$ tercil, 24 a 56 anos), situação conjugal (com ou sem parceiro), período de estudo (diurno e noturno) e áreas de estudo, categorizadas conforme as áreas de conhecimento propostas pela Coordenação de Aperfeiçoamento de Pessoal de Nível Superior ${ }^{15}$, com base os cursos da instituição (Ciências Exatas e da Terra, Ciências Biológicas, Engenharias, Ciências Agrárias, Ciências Sociais e Aplicadas, Ciências Humanas, Ciências da Saúde, Letras e Artes).

As informações foram tabuladas no software EpiData 3.1 e as análises conduzidas no programa $S P S S^{\circledast}$, versão 24.0. As análises descritivas incluíram as frequências absolutas e relativas, média e desvio padrão (DP), mínimo e máximo valores. O teste do qui-quadrado para tendência linear foi usado para comparar as prevalências de prática de cada modalidade entre os anos dos inquéritos de modo geral e estratificados pelas categorias das variáveis sociodemográficas e de vínculo com a universidade. As associações foram significativas para um nível de significância de 5\%.

\section{Resultados}

Participaram do estudo 1.084, 1.085 e 1.041 universitários, nos anos de 2010, 2012 e 2014, respectivamente. A média de idade em cada ano de inquérito foi de 23,55 anos (DP = 5,25; de 17 a 52) em 2010, 23,99 anos (DP = 6,01; de 17 a 54) em 2012 e 23,65 anos (DP = 5,77; de 17 a 57) em 2014. A descrição sociodemográfica e de vínculo com a universidade é apresentada na Tabela 1. Nos três anos de inquérito, a maior parte dos estudantes foram do sexo feminino e do período de estudo diurno. Houve predomínio de universitários da área das Ciências Sociais e Aplicada.

Em relação às prevalências de práticas de atividades físicas no lazer, a modalidade mais praticada nos três inquéritos foi a musculação, seguida do futebol. As modalidades de corrida $(2010=6,9 \% ; 2012=8,8 \% ; 2014$ $=11,0 \%)$ e de lutas ou artes marciais $(2010=4,2 \%$; $2012=4,5 \% ; 2014=7,6 \%)$ tiveram aumentos significativos na sua prática ao longo dos inquéritos. Em contrapartida, houve a diminuição da prática da ginástica em geral com uso de pesos e trampolins $(2010=3,7 \%$; $2012=1,9 \% ; 2014=1,4 \% ; p=0,001$ ). 
Tabela 1 - Descrição da amostra dos universitários de acordo com as variáveis sociodemográficas e de vínculo com a universidade. Bahia. 2010, 2012 e 2014.

\begin{tabular}{lccc}
\hline Variáveis & 2010 & 2012 & 2014 \\
$\mathrm{n}(\%)$ & $\mathrm{n}(\%)$ & $\mathrm{n}(\%)$ \\
\hline Sexo & & & \\
$\quad$ Masculino & $491(45,3)$ & $489(45,1)$ & $494(47,5)$ \\
$\quad$ Feminino & $592(54,7)$ & $595(54,9)$ & $547(52,5)$ \\
Faixa etária & & & \\
1 $^{\circ}$ tercil & $285(26,7)$ & $304(28,3)$ & $322(31,2)$ \\
$2^{\circ}$ tercil & $400(37,4)$ & $358(33,3)$ & $352(34,1)$ \\
$3^{\circ}$ tercil & $384(35,9)$ & $412(38,4)$ & $357(34,7)$ \\
Período de estudo & & & \\
Diurno & $735(67,8)$ & $731(67,4)$ & $747(71,8)$ \\
Noturno & $349(32,2)$ & $354(32,6)$ & $294(28,2)$ \\
Área de estudo & & & \\
Agrarias & $95(8,8)$ & $91(8,4)$ & $80(7,7)$ \\
Exatas e da terra & $181(16,7)$ & $190(17,5)$ & $168(16,1)$ \\
Sociais e aplicada & $290(26,8)$ & $237(21,8)$ & $219(21,0)$ \\
Biológicas & $76(7,0)$ & $75(6,9)$ & $84(8,1)$ \\
Engenharias & $38(3,5)$ & $92(8,5)$ & $138(13,3)$ \\
Humanas & $145(13,4)$ & $164(15,1)$ & $143(13,7)$ \\
Saúde & $126(11,6)$ & $113(10,4)$ & $108(10,4)$ \\
Letras e artes & $133(12,3)$ & $123(11,3)$ & $101(9,7)$ \\
\hline
\end{tabular}

Inquérito de $2010=1^{\circ}$ tercil, 17 a 20 anos; $2^{\circ}$ tercil, 21 a 23 anos; $3^{\circ}$ tercil, 24 a 52 anos; Inquérito de $2012=1^{\circ}$ tercil, 17 a 20 anos; $2^{\circ}$ tercil, 21 a 23 anos; $3^{\circ}$ tercil, 24 a 54 anos; Inquérito de $2014=1^{\circ}$ tercil, 17 a 20 anos; $2^{\circ}$ tercil, 21 a 23 anos; $3^{\circ}$ tercil, 24 a 56 anos.

Tabela 2 - Comparação das prevalências de práticas de atividade física no lazer entre os anos dos inquéritos em universitários. Bahia. 2010, 2012 e 2014.

\begin{tabular}{lcccc}
\hline Variáveis & $\begin{array}{c}2010^{*} \\
\mathrm{n}(\%)\end{array}$ & $\begin{array}{c}2012 \\
\mathrm{n}(\%)\end{array}$ & $\begin{array}{c}2014 \\
\mathrm{n}(\%)\end{array}$ & $\mathrm{p}^{* *}$ \\
\hline Futebol & $173(16,3)$ & $171(16,0$ & $171(16,0)$ & 0,857 \\
Voleibol & $15(1,4)$ & $19(1,8$ & $25(2,4)$ & 0,087 \\
Basquetebol & $9(0,8)$ & $12(1,1$ & $8(0,8)$ & 0,870 \\
Handebol & $10(0,9)$ & $12(1,1$ & $3(0,3)$ & 0,095 \\
Ginástica em geral com uso & $39(3,7)$ & $20(1,9$ & $14(1,4)$ & 0,001 \\
de pesos e trampolins & $73(6,9)$ & $94(8,8$ & $113(11,0)$ & 0,001 \\
Corrida & $45(4,2)$ & $59(5,5$ & $41(4,0)$ & 0,787 \\
Ginástica aeróbica & $52(4,9)$ & $44(4,1$ & $53(5,2)$ & 0,799 \\
Ciclismo & $45(4,2)$ & $48(4,5$ & $78(7,6)$ & 0,001 \\
Lutas ou artes marciais & $5(0,5)$ & $7(0,7$ & $5(0,5)$ & 0,960 \\
Tênis & $44(4,2)$ & $64(6,0$ & $67(6,5)$ & 0,018 \\
Corrida na esteira & $31(2,9)$ & $41(3,8$ & $42(4,1)$ & 0,155 \\
Caminhada na esteira & $4(0,4)$ & $6(0,6$ & $3(0,3)$ & 0,766 \\
Hidroginástica & $23(2,3 \mathrm{v}$ & $14(1,3$ & $20(1,9)$ & 0,688 \\
Surfe & $28(2,6)$ & $21(2,0$ & $18(1,7)$ & 0,156 \\
Natação & $222(20,9)$ & $261(24,3$ & $241(23,4)$ & 0,174 \\
Musculação & $76(7,2)$ & $87(8,1$ & $88(8,6)$ & 0,244 \\
Caminhada & & & & \\
\hline
\end{tabular}

*Prevalências apresentadas por Sousa ${ }^{5} ;{ }^{* *}$ Valor de $\mathrm{p}$ do teste qui-quadrado para tendência linear.
A Tabela 3 apresenta as prevalências das modalidades esportivas de quadra e lutas ou artes marciais de acordo com as variáveis sociodemográficas. Em relação à modalidade do voleibol foi possível observar o aumento ao longo dos inquéritos entre os estudantes do segundo tercil de idade $(2010=1,3 \% ; 2012=2,2 \%$; $2014=3,4 \%)$, como também entre os universitários da área das Ciências Humanas entre os anos de 2010 $(1,0 \%)$ e 2014 (3,6\%).

A prática de lutas ou artes marciais apresentou aumento significativo nesse período, entre os estudantes do sexo masculino $(2010=5,6 \% ; 2012=6,4 \% ; 2014$ $=10,5 \%)$ e aqueles do terceiro tercil de idade $(2010=$ 3,5\%; $2012=3 \% ; 2014=8,8 \%)$. Também houve aumento na prática das lutas ou artes marciais em ambos os períodos de estudo, diurno $(2010=5,2 \% ; 2012=5,4 \%$; $2014=8,1 \%)$ e noturno $(2010=2,3 \% ; 2012=2,6 \%$; $2014=6,2 \%)$, e entre os universitários das áreas de estudo das Ciências Sociais e Aplicada, passando de 3,2\% em 2010 para 10,1\% em 2014, e da área das Ciências Humanas $(2010=1,4 \% ; 2012=3,7 \% ; 2014=6,9 \%)$.

Em relação às modalidades de corridas (Tabela 4), as prevalências aumentaram entre as mulheres e os estudantes do período diurno entre os três anos dos inquéritos. Entre os universitários do primeiro $(2010=$ $5,7 \% ; 2012=7,6 \% ; 2014=11,6 \%)$ e segundo $(2010$ $=5,6 \% ; 2012=9,2 \% ; 2014=10,3 \%)$ tercis de idade, houve aumento da prevalência da prática de corrida em locais abertos e também entre os estudantes da área das Ciências Exatas e da Terra $(2010=4,5 \% ; 2012=5,9 \%$; $2014=12,3 \%)$. A prática de caminhada em locais abertos também apresentou aumento entre os universitários que estavam no primeiro tercil da faixa etária $(2010=$ $5,4 \% ; 2012=6,3 ; 2014=11 \%$ ).

A Tabela 5 apresenta as prevalências de prática das modalidades aquáticas, musculação e ginástica em geral com uso de pesos e trampolins. Foi possível observar que houve diminuição, entre os anos dos inquéritos, na prática de natação entre os estudantes do terceiro tercil de idade $(2010=3,5 \% ; 2012=0,5 \% ; 2014=$ $1,1 \%)$. Na modalidade de musculação houve aumento da prevalência da prática entre as mulheres (2010 = 14,5\%; $2012=19,8 \% ; 2014=20,1 \%)$. Em contrapartida ocorreu a diminuição da prática de ginástica entre os anos dos inquéritos para as mulheres $(2010=4,7 \%$; $2012=2,7 \% ; 2014=1,5 \%)$, os universitários do segundo $(2010=4,8 \% ; 2012=2,0 \% ; 2014=1,4 \%)$ e terceiro tercis de idade, e entre os estudantes do período de estudo diurno $(2010=3,6 \% ; 2012=2,5 \% ; 2014=1,1 \%)$. 
Tabela 3 - Comparação das prevalências das modalidades esportivas de quadra e lutas ou artes marciais entre os anos dos inquéritos, de acordo com as variáveis sociodemográficas e de vínculo com a universidade. Bahia. 2010, 2012 e 2014.

\begin{tabular}{|c|c|c|c|c|c|c|c|c|c|}
\hline \multirow[b]{2}{*}{ Variáveis } & \multicolumn{3}{|c|}{ Futebol } & \multicolumn{3}{|c|}{ Voleibol } & \multicolumn{3}{|c|}{ Basquetebol } \\
\hline & $\begin{array}{c}2010^{*} \\
\%\end{array}$ & $\begin{array}{c}2012 \\
\%\end{array}$ & $\begin{array}{c}2014 \\
\%\end{array}$ & $\begin{array}{c}2010^{*} \\
\%\end{array}$ & $\begin{array}{c}2012 \\
\%\end{array}$ & $\begin{array}{c}2014 \\
\%\end{array}$ & $\begin{array}{c}2010^{*} \\
\%\end{array}$ & $\begin{array}{c}2012 \\
\%\end{array}$ & $\begin{array}{c}2014 \\
\%\end{array}$ \\
\hline \multicolumn{10}{|l|}{ Sexo } \\
\hline Masculino & 34,5 & 34,1 & 33,3 & 2,3 & 2,5 & 4,3 & 1,7 & 2,3 & 1,6 \\
\hline Feminino & 1,1 & 1,2 & 1,7 & 0,7 & 1,2 & 5,0 & 0,2 & 0,2 & - \\
\hline \multicolumn{10}{|l|}{ Faixa etária } \\
\hline $1^{\circ}$ tercil & 16,8 & 15,5 & 14,8 & 2,5 & 2,6 & 3,1 & 0,4 & 0,7 & 0,6 \\
\hline $2^{\circ}$ tercil & 17,3 & 16,5 & 17,1 & 1,3 & 2,2 & $3,4^{* *}$ & 1,3 & 2,0 & 1,1 \\
\hline $3^{\circ}$ tercil & 15,7 & 16,0 & 17,9 & 0,8 & 0,7 & 0,9 & 0,8 & 0,7 & 0,6 \\
\hline \multicolumn{10}{|l|}{ Período de estudo } \\
\hline Diurno & 15,3 & 15,7 & 15,0 & 1,4 & 1,8 & 2,7 & 0,7 & 0,8 & 0,9 \\
\hline Noturno & 18,4 & 16,4 & 20,7 & 1,5 & 1,7 & 1,7 & 1,2 & 1,7 & 0,3 \\
\hline \multicolumn{10}{|l|}{ Área de estudo } \\
\hline Agrarias & 18,5 & 12,4 & 15,0 & 1,1 & 1,1 & 1,3 & 1,1 & - & - \\
\hline Exatas e da terra & 24,3 & 19,4 & 21,5 & 2,8 & 3,8 & 4,3 & 1,1 & 2,7 & 1,8 \\
\hline Sociais e aplicada & 20,8 & 17,9 & 17,1 & 1,4 & 1,3 & 1,8 & 0,7 & 0,4 & 0,5 \\
\hline Biológicas & 6,7 & 12,0 & 10,7 & - & 5,3 & - & - & - & - \\
\hline Engenharias & 28,9 & 30,4 & 26,1 & - & 2,2 & 1,1 & - & - & 1,4 \\
\hline Humanas & 9,2 & 8,7 & 15,7 & 1,0 & - & $3,6^{* *}$ & 1,4 & 1,2 & 0,7 \\
\hline Saúde & 14,5 & 21,2 & 14,0 & 3,2 & 1,8 & 4,7 & 1,6 & 1,8 & 0,9 \\
\hline Letras e artes & 5,5 & 5,7 & 5,0 & - & - & 1,0 & - & 1,6 & - \\
\hline \multirow[b]{2}{*}{ Variáveis } & \multicolumn{3}{|c|}{ Handebol } & \multicolumn{3}{|c|}{ Tênis de quadra } & \multicolumn{3}{|c|}{ Lutas ou artes marciais } \\
\hline & $\begin{array}{c}2010^{*} \\
\%\end{array}$ & $\begin{array}{c}2012 \\
\%\end{array}$ & $\begin{array}{c}2014 \\
\%\end{array}$ & $\begin{array}{c}2010^{*} \\
\%\end{array}$ & $\begin{array}{c}2012 \\
\%\end{array}$ & $\begin{array}{c}2014 \\
\%\end{array}$ & $\begin{array}{c}2010^{*} \\
\%\end{array}$ & $\begin{array}{c}2012 \\
\%\end{array}$ & $\begin{array}{c}2014 \\
\%\end{array}$ \\
\hline \multicolumn{10}{|l|}{ Sexo } \\
\hline Masculino & 0,4 & 1,7 & 0,4 & 0,4 & 1,0 & 1,0 & 5,6 & 6,4 & $10,5^{* *}$ \\
\hline Feminino & 1,4 & 0,7 & $0,2^{* *}$ & 0,5 & 0,3 & - & 3,1 & 2,9 & 5,0 \\
\hline \multicolumn{10}{|l|}{ Faixa etária } \\
\hline $1^{\circ}$ tercil & 2,5 & 2,0 & 0,6 & 0,7 & 1,0 & - & 3,9 & 5,6 & 6,0 \\
\hline $2^{\circ}$ tercil & 0,3 & 1,4 & - & 0,3 & 0,3 & 1,0 & 5,1 & 5,0 & 8,0 \\
\hline $3^{\circ}$ tercil & 0,5 & 0,2 & 0,3 & 0,5 & 0,7 & 0,3 & 3,5 & 3,0 & $8,8^{* *}$ \\
\hline \multicolumn{10}{|l|}{ Período de estudo } \\
\hline Diurno & 1,0 & 1,4 & 0,4 & 0,6 & 0,7 & 0,5 & 5,2 & 5,4 & $8,1^{* *}$ \\
\hline Noturno & 0,9 & 0,6 & - & 0,3 & 0,6 & 0,4 & 2,3 & 2,6 & $6,2^{* *}$ \\
\hline \multicolumn{10}{|l|}{ Área de estudo } \\
\hline Agrarias & 1,1 & - & - & - & 1,1 & 1,3 & 4,3 & 4,5 & 1,3 \\
\hline Exatas e da terra & 1,1 & 1,6 & - & - & 1,1 & 1,2 & 5,6 & 5,4 & 7,4 \\
\hline Sociais e aplicada & 1,1 & 0,4 & 0,5 & 0,7 & 0,4 & - & 3,2 & 2,6 & $10,1^{* *}$ \\
\hline Biológicas & - & 1,3 & - & 1,3 & - & - & 5,3 & 5,3 & 7,1 \\
\hline Engenharias & - & 5,4 & 0,7 & 2,6 & 1,1 & 1,4 & 10,5 & 6,5 & 10,9 \\
\hline Humanas & 0,7 & 0,7 & - & - & 0,6 & - & 1,4 & 3,7 & $6,9^{* *}$ \\
\hline Saúde & 2,4 & 1,8 & 0,9 & 0,8 & - & - & 4,0 & 8,0 & 9,3 \\
\hline Letras e artes & - & - & - & - & 0,8 & - & 5,6 & 2,3 & 3,0 \\
\hline
\end{tabular}

*Prevalências apresentadas por Sousa ${ }^{5}$;*Indica que houve valor de p do teste qui-quadrado para tendência linear $<0,05$ na comparação das prevalências entre os inquéritos; -Valores de prevalências não estimados devido à ausência de resposta. 
Tabela 4 - Comparação das prevalências das modalidades aeróbias entre os anos dos inquéritos, de acordo com as variáveis sociodemográficas e de vínculo com a universidade. Bahia. 2010, 2012 e 2014.

\begin{tabular}{|c|c|c|c|c|c|c|c|c|c|}
\hline \multirow[b]{2}{*}{ Variáveis } & \multicolumn{3}{|c|}{ Corrida em locais abertos } & \multicolumn{3}{|c|}{ Corrida em esteira } & \multicolumn{3}{|c|}{ Ciclismo } \\
\hline & $\begin{array}{c}2010^{*} \\
\%\end{array}$ & $\begin{array}{c}2012 \\
\%\end{array}$ & $\begin{array}{c}2014 \\
\%\end{array}$ & $\begin{array}{c}2010^{*} \\
\%\end{array}$ & $\begin{array}{c}2012 \\
\%\end{array}$ & $\begin{array}{c}2014 \\
\%\end{array}$ & $\begin{array}{c}2010^{*} \\
\%\end{array}$ & $\begin{array}{c}2012 \\
\%\end{array}$ & $\begin{array}{c}2014 \\
\%\end{array}$ \\
\hline \multicolumn{10}{|l|}{ Sexo } \\
\hline Masculino & 10,6 & 12,2 & 13,8 & 5,0 & 5,4 & 5,1 & 5,4 & 4,2 & 7,0 \\
\hline Feminino & 4,2 & 5,6 & $8,5^{* *}$ & 3,5 & 6,4 & $7,7^{* *}$ & 4,5 & 4,2 & 3,5 \\
\hline \multicolumn{10}{|l|}{ Faixa etária } \\
\hline $1^{\circ}$ tercil & 5,7 & 7,6 & $11,6^{* *}$ & 5,0 & 5,3 & 6,3 & 3,2 & 2,3 & 3,8 \\
\hline $2^{\circ}$ tercil & 5,6 & 9,2 & $10,3^{* *}$ & 4,8 & 5,9 & 6,9 & 4,8 & 1,5 & 4,3 \\
\hline $3^{\circ}$ tercil & 9,4 & 9,5 & 10,8 & 3,0 & 6,5 & 6,0 & 6,5 & 5,2 & 7,1 \\
\hline \multicolumn{10}{|l|}{ Período de estudo } \\
\hline Diurno & 7,2 & 9,2 & $11,8^{* *}$ & 4,5 & 6,6 & $7,4^{* * *}$ & 4,0 & 4,0 & 4,5 \\
\hline Noturno & 6,1 & 7,8 & 9,0 & 3,5 & 4,6 & 4,1 & 6,7 & 4,3 & 6,9 \\
\hline \multicolumn{10}{|l|}{ Área de estudo } \\
\hline Agrarias & 6,5 & 7,9 & 10,0 & 4,3 & 3,4 & 5,0 & 5,4 & 1,1 & 7,5 \\
\hline Exatas e da terra & 4,5 & 5,9 & $12,3^{* *}$ & 1,1 & 4,3 & 4,3 & 5,1 & 7,0 & 9,8 \\
\hline Sociais e aplicada & 8,8 & 7,3 & 13,4 & 5,6 & 7,7 & 7,8 & 5,3 & 5,6 & 3,2 \\
\hline Biológicas & 12,0 & 8,0 & 9,5 & 4,0 & 8,0 & 9,5 & 8,0 & 8,0 & 6,0 \\
\hline Engenharias & 5,3 & 9,8 & 11,6 & 5,3 & 5,4 & 11,6 & - & - & 2,9 \\
\hline Humanas & 7,6 & 5,6 & 7,9 & 3,5 & 4,3 & 4,3 & 4,3 & 3,7 & 7,1 \\
\hline Saúde & 6,5 & 21,2 & $15,0^{* * *}$ & 6,5 & 9,7 & 1,9 & 5,6 & 3,5 & 1,9 \\
\hline Letras e artes & 3,1 & 9,0 & 5,0 & 3,1 & 4,9 & 7,0 & 3,1 & 2,5 & 3,0 \\
\hline \multirow{3}{*}{ Variáveis } & \multicolumn{3}{|c|}{ Caminhada em locais abertos } & \multicolumn{3}{|c|}{ Caminhada em esteira } & \multicolumn{3}{|c|}{ Ginástica aeróbica } \\
\hline & $2010^{*}$ & 2012 & 2014 & $2010^{*}$ & 2012 & 2014 & $2010^{*}$ & 2012 & 2014 \\
\hline & $\%$ & $\%$ & $\%$ & $\%$ & $\%$ & $\%$ & $\%$ & $\%$ & $\%$ \\
\hline \multicolumn{10}{|l|}{ Sexo } \\
\hline Masculino & 6,9 & 6,4 & 8,0 & 1,7 & 2,5 & 3,5 & 2,3 & 2,3 & 1,6 \\
\hline Feminino & 7,4 & 9,3 & 9,0 & 4,0 & 4,9 & 4,6 & 5,9 & 8,1 & 6,1 \\
\hline \multicolumn{10}{|l|}{ Faixa etária } \\
\hline $1^{\circ}$ tercil & 5,4 & 6,3 & $11,0^{* *}$ & 3,6 & 2,6 & 4,4 & 2,5 & 6,3 & 4,7 \\
\hline $2^{\circ}$ tercil & 6,1 & 6,4 & 6,3 & 3,0 & 4,5 & 4,0 & 5,3 & 5,6 & 4,6 \\
\hline $3^{\circ}$ tercil & 8,9 & 11,0 & 8,5 & 2,4 & 4,2 & 3,7 & 4,6 & 4,7 & 2,8 \\
\hline \multicolumn{10}{|l|}{ Período de estudo } \\
\hline Diurno & 7,3 & 6,5 & 8,4 & 3,2 & 4,1 & 3,8 & 4,6 & 5,9 & 4,2 \\
\hline Noturno & 7,0 & 11,5 & 9,0 & 2,3 & 3,2 & 4,8 & 3,5 & 4,6 & 3,4 \\
\hline \multicolumn{10}{|l|}{ Área de estudo } \\
\hline Agrarias & 5,4 & 6,7 & 11,3 & 3,3 & 3,4 & 5,0 & 3,3 & 6,7 & 1,3 \\
\hline Exatas e da terra & 6,8 & 8,1 & 9,2 & 1,1 & 1,1 & 2,5 & 1,7 & 4,3 & 3,7 \\
\hline Sociais e aplicada & 6,7 & 11,1 & 9,2 & 2,5 & 3,0 & 5,1 & 4,2 & 6,4 & 4,6 \\
\hline Biológicas & 9,3 & 4,0 & 9,5 & 5,3 & 6,7 & 7,1 & 5,3 & 6,7 & 4,8 \\
\hline Engenharias & 5,3 & 4,3 & 7,2 & 2,6 & 4,3 & 2,9 & 7,9 & 5,4 & 2,2 \\
\hline Humanas & 9,2 & 9,3 & 6,4 & 2,8 & 4,3 & 3,0 & 3,5 & 4,3 & 4,3 \\
\hline Saúde & 6,5 & 1,8 & 8,4 & 6,5 & 8,0 & 4,7 & 8,1 & 7,1 & 8,4 \\
\hline Letras e artes & 7,8 & 13,1 & 8,0 & 1,6 & 3,3 & 1,0 & 3,9 & 4,1 & 2,0 \\
\hline
\end{tabular}

*Prevalências apresentadas por Sousa ${ }^{5}$;*Indica que houve valor de p do teste qui-quadrado para tendência linear $<0,05$ na comparação das prevalências entre os inquéritos; -Valores de prevalências não estimados devido à ausência de resposta. 
Tabela 5 - Comparação das prevalências das modalidades aquáticas, musculação e ginástica entre os anos dos inquéritos, de acordo com as variáveis sociodemográficas e de vínculo com a universidade. Bahia. 2010, 2012 e 2014.

\begin{tabular}{|c|c|c|c|c|c|c|c|c|c|c|c|c|c|c|c|}
\hline \multirow{2}{*}{ Variáveis } & \multicolumn{3}{|c|}{ Hidroginástica } & \multicolumn{3}{|c|}{ Surfe } & \multicolumn{3}{|c|}{ Natação } & \multicolumn{3}{|c|}{ Musculação } & \multicolumn{3}{|c|}{$\begin{array}{l}\text { Ginástica em geral com } \\
\text { uso de pesos e trampolins }\end{array}$} \\
\hline & $\begin{array}{l}2010^{*} \\
\%\end{array}$ & $\begin{array}{c}2012 \\
\%\end{array}$ & $\begin{array}{c}2014 \\
\%\end{array}$ & $\begin{array}{c}2010^{*} \\
\%\end{array}$ & $\begin{array}{c}2012 \\
\%\end{array}$ & $\begin{array}{c}2014 \\
\%\end{array}$ & $\begin{array}{c}2010^{*} \\
\%\end{array}$ & $\begin{array}{c}2012 \\
\%\end{array}$ & $\begin{array}{c}2014 \\
\%\end{array}$ & $\begin{array}{l}2010^{*} \\
\%\end{array}$ & $\begin{array}{c}2012 \\
\%\end{array}$ & $\begin{array}{c}2014 \\
\%\end{array}$ & $\begin{array}{c}2010^{*} \\
\%\end{array}$ & $\begin{array}{c}2012 \\
\%\end{array}$ & $\begin{array}{c}2014 \\
\%\end{array}$ \\
\hline \multicolumn{16}{|l|}{ Sexo } \\
\hline Masculino & 0,2 & - & 0,2 & 4,0 & 2,5 & 3,3 & 3,5 & 3,1 & 2,3 & 28,5 & 29,9 & 27,1 & 2,5 & 0,8 & 1,2 \\
\hline Feminino & 0,5 & 1,0 & 0,4 & 0,7 & 0,3 & 0,7 & 1,9 & 1,0 & 1,3 & 14,5 & 19,8 & $20,1^{\text {*** }}$ & 4,7 & 2,7 & $1,5^{* *}$ \\
\hline \multicolumn{16}{|l|}{ Faixa etária } \\
\hline $1^{o}$ tercil & - & 0,7 & - & 1,4 & 1,7 & 1,6 & 2,9 & 3,6 & 1,3 & 22,9 & 24,8 & 26,7 & 3,6 & 2,0 & $0,3^{* *}$ \\
\hline $2^{\circ}$ tercil & - & 0,3 & 0,3 & 1,5 & 0,8 & 1,7 & 1,8 & 2,0 & 2,9 & 24,4 & 29,4 & 25,1 & 4,8 & 2,0 & $1,4^{* *}$ \\
\hline $3^{\circ}$ tercil & 1,1 & 0,7 & 0,6 & 3,5 & 1,5 & 2,6 & 3,5 & 0,5 & $1,1^{\text {** }}$ & 16,4 & 19,5 & 18,2 & 2,7 & 1,7 & 2,3 \\
\hline \multicolumn{16}{|l|}{ Período de estudo } \\
\hline Diurno & 0,3 & 0,6 & 0,1 & 2,4 & 1,7 & 2,6 & 3,1 & 2,2 & 2,0 & 24,4 & 27,9 & 25,3 & 3,6 & 2,5 & $1,1^{* *}$ \\
\hline Noturno & 0,6 & 0,6 & 0,7 & 1,8 & 0,6 & 0,3 & 1,8 & 1,4 & 1,0 & 13,7 & 17,0 & 18,6 & 3,8 & 0,6 & 2,1 \\
\hline \multicolumn{16}{|l|}{ Área de estudo } \\
\hline Agrarias & - & 1,1 & - & $5,4^{* *}$ & - & - & 5,4 & 2,2 & 3,8 & 22,8 & 28,1 & 25,0 & 3,3 & 1,1 & 1,3 \\
\hline Exatas e da terra & - & 1,1 & - & 2,8 & 0,5 & 2,5 & 2,8 & 2,2 & 1,8 & 16,9 & 17,7 & 22,1 & 2,8 & 1,6 & 1,2 \\
\hline Sociais e aplicada & 0,4 & 0,9 & 0,5 & 2,6 & 0,4 & 1,8 & 2,1 & 2,1 & 1,4 & 19,7 & 24,4 & 20,7 & 4,2 & 3,0 & 1,4 \\
\hline Biológicas & - & - & - & 2,7 & 1,3 & 2,4 & 2,7 & - & 1,2 & 33,3 & 30,7 & 26,2 & 4,0 & 2,7 & 1,2 \\
\hline Engenharias & - & - & - & 2,6 & 3,3 & 2,9 & 2,6 & 3,3 & 2,9 & 28,9 & 33,7 & 33,3 & - & - & - \\
\hline Humanas & 1,4 & - & 0,7 & - & - & 0,7 & 1,4 & - & 1,4 & 14,1 & 19,9 & 15,0 & 4,2 & 1,2 & 2,1 \\
\hline Saúde & - & - & 0,9 & 2,4 & 5,3 & 3,7 & 4,0 & - & 0,9 & 30,6 & 37,2 & 35,5 & 6,6 & 0,9 & 3,7 \\
\hline Letras e artes & 0,8 & 0,8 & - & - & 1,6 & 1,0 & 1,6 & 5,7 & 1,0 & 16,4 & 14,8 & 13,0 & 1,6 & 3,3 & - \\
\hline
\end{tabular}

*Prevalências apresentadas por Sousa ${ }^{5}$; **Indica que houve valor de $\mathrm{p}$ do teste qui-quadrado para tendência linear $<0,05$ na comparação das prevalências entre os inquéritos; -Valores de prevalências não estimados devido à ausência de resposta.

\section{Discussão}

Neste estudo as modalidades mais praticadas pelos universitários em suas atividades físicas de lazer foram a musculação e o futebol, já as modalidades de corrida e lutas ou artes marciais tiveram um aumento significativo ao longo dos três inquéritos. Entretanto, houve uma diminuição da prática de ginástica em geral com uso de pesos e trampolins. Notou-se que, para algumas categorias sociodemográficas e de vínculo com a universidade, houve mudanças ao longo dos anos nas prevalências de prática de atividades físicas no lazer em diferentes modalidades.

Os resultados deste estudo mostraram que as modalidades que predominaram, musculação e futebol, foram semelhantes a de outro estudo realizado no estado da Bahia, porém, conduzido apenas com estudantes de Educação Física, que mostrou como atividades físicas no lazer mais praticadas a ginástica/musculação $(25,7 \%)$ e os esportes coletivos $(23,8 \%)^{16}$. Além disso, as modalidades que predominaram no inquérito de 2010, conforme apresentado por Sousa ${ }^{5}$, mantiveram- -se semelhantes nos outros dois anos de inquéritos. É importante considerar que a prática da musculação representa a principal modalidade de atividade física no lazer de adultos brasileiros ${ }^{17}$, e neste estudo houve o aumento da prática entre as mulheres. $\mathrm{O}$ que pode demonstrar nesse grupo a relevância atribuída à prática de exercícios resistidos como promotor de benefícios para a saúde ${ }^{18}$, mas, também em virtude da preocupação do público jovem com aspectos estéticos, como manutenção da silhueta corporal ${ }^{19}$.

Neste estudo, verificou-se que a prática de lutas ou artes marciais obteve aumento significativo entre os anos, para os estudantes do sexo masculino, idade avançada, que estudavam no período diurno e noturno, e das áreas das Ciências Sociais e Aplicada e Ciências Humanas. A transposição das modalidades de lutas como esporte de massa que ocorreram ao longo dos últimos anos podem representar um incentivo a adesão à prática ${ }^{20}$, especialmente com o advento das artes marciais mistas e os eventos competitivos organizados para essa modalidade como espetáculo, disponibiliza- 
dos tanto em mídias televisas fechadas quanto abertas, contribuem para o favorecimento ao ingresso nessa modalidade. Assim como as especificidades culturais do estado da Bahia, que oportunizam a presença da capoeira no cenário social e podem favorecer a evolução da prática dessa modalidade ${ }^{21}$.

Em contrapartida, houve a diminuição da prática de ginástica em geral, realizada com usos de pesos e trampolins, entre as mulheres. Possivelmente, essa diminuição esteja atrelada a ênfase no cenário atual para outros tipos de atividades ginásticas, como os treinamentos funcionais $^{22}$ e o Crossfit , ambos voltados para a manutenção dos níveis de aptidão física para a saúde. Para o ingresso nessas modalidades há a necessidade de recursos finan$\operatorname{ceiros}^{23}$, deste modo isso pode ocasionar no processo natural de escolha entre as atividades, resultando na descontinuidade, por exemplo da ginástica em geral com uso de pesos e trampolins, e adesão em outras ${ }^{24}$.

De modo geral, este estudo mostrou que o futebol foi uma das principais modalidades praticadas, contudo, juntamente com outros esportes coletivos, houve a estabilidade da prática ao longo dos inquéritos. Em outro estudo, porém, realizado em Campo Grande, Mato Grosso Sul, futsal (15\%) e basquete (13\%) foram as principais modalidades relatadas ${ }^{25}$. Já neste estudo, houve o aumento da proporção de praticantes de voleibol na faixa de idade de 21 a 23 anos e entre os universitários pertencentes à área das Ciências Humanas. O voleibol é um dos esportes que mais expandiu ao longo dos anos no Brasil, especialmente com a espetacularização da modalidade, o que possivelmente pode influenciar os universitários mais joven ${ }^{26}$.

Já em relação à atividade física no lazer de predominância aeróbia, como a corrida em locais abertos, observou-se que essa foi uma das principais práticas entre as universitárias. A caminhada ${ }^{27-29}$ e corrida $^{27}$ foram relatadas em outros estudos como sendo as principais modalidades praticadas por mulheres. As práticas aeróbias realizadas no lazer, como a caminhada, podem possibilitar menores riscos de fraturas ${ }^{30}$ e a obtenção de benefícios em níveis metabólicos e musculares de mulheres na meia idade e idosas, deste modo a prática em idades iniciais poderá favorecer o envelhecimento saudável, além disso, representam atividades de maior acessibilidade, que assim oportunizam a realização de forma regular e com maior autonomia das praticantes.

Em relação à modalidade de corrida em locais abertos, observou-se entre os inquéritos, o aumento da quantidade de praticantes entre os universitários da área das Ciências da Saúde. Os universitários de cursos dessa área, como aqueles vinculados à Educação Física, demonstram maior envolvimento em atividades físicas de lazer de modo geral ${ }^{3}$. Além disso, em outra pesquisa, os estudantes da área das Ciências da Saúde apresentaram maior envolvimento com a prática de exercícios resistidos (musculação) ${ }^{5}$. Espera-se o maior envolvimento de universitários dessa área com práticas motoras durante o tempo livre, como as corridas, em virtude dos conteúdos que normalmente são discutidos nos componentes curriculares, que podem favorecer a ampliação do conhecimento sobre a importância dessa conduta, bem como a atribuição de valor significativo dentre os hábitos de vida.

Neste estudo houve a diminuição da prática de natação entre os estudantes de idade avançada. Em pesquisa realizada por Oliveira e colaboradores ${ }^{25}$, a prática da modalidade natação foi de apenas $0,7 \%$ entre os universitários. A diminuição da prática nessa modalidade, pode estar relacionada à necessidade de espaços que possibilitam a adesão, que normalmente não são acessíveis a todos, pois requer suporte financeiro e assim podem ser substituídas por outras obrigações profissionais ou familiares ao longo do tempo. Deste modo, caso as universidades tenham esse equipamento, a oferta de programas ou projetos voltados às atividades aquáticas poderá representar uma importante oportunidade de prática.

Embora este estudo apresente pontos fortes como a realização de procedimento metodológico robusto e a realização de processo de seleção aleatória simples para a participação na pesquisa, as seguintes limitações podem ser citadas: uso de questionário para a mensuração das informações que pode ocasionar em viés de informação, com a superestimação das respostas positivas, em virtude do conhecimento da relevância da prática no lazer como benéfica para a saúde e o surgimento de modalidades ao longo dos anos, que não estavam contempladas dentre as opções e assim dificultar a equiparação com outras atividades, contudo, o presente instrumento apresenta níveis satisfatórios de reprodutibilidade ${ }^{14}$.

O entendimento sobre o perfil de prática de atividades físicas de lazer ao longo do tempo em universitários, permite que a instituição participante deste estudo realize ações específicas, por meio de projetos e programas destinados a prática das modalidades de maior preferência. Além disso, as informações deste estudo contribuem para que outras atividades possam ser implementadas, como forma de aprendizado de novas 
experiências motoras ou como advento de promoção de saúde com foco em grupos de maior vulnerabilidade em relação a prática no tempo livre.

Conclui-se que a prática da musculação foi a modalidade preferida pelos universitários e que ao longo dos anos houve de modo geral a estabilização da quantidade de praticantes de todas as atividades, exceto para lutas ou artes marciais e corridas, as quais aumentaram, e para ginástica em geral com uso de pesos e trampolins, que diminuíram no período de quatro anos. Houve modificações nas proporções de praticantes das modalidades em diferentes categorias das variáveis sociodemográficas e de vínculo com a universidade, especialmente para as lutas ou artes marciais e corridas em locais abertos.

\section{Declaração de conflitos de interesse}

Os autores declaram não haver conflito de interesses.

\section{Financiamento}

Esta pesquisa apresentou financiamento em forma de bolsa de estudos durante o mestrado e doutorado do primeiro autor deste artigo na realização dos inquéritos de 2010 e 2012 . O inquérito de 2014 foi realizado com recursos próprios do coordenador da pesquisa.

\section{Contribuição dos autores}

Sousa TF, elaborou e coordena o estudo MONISA, realizou as análises estatísticas e redação do artigo. Franco DC, desenvolveu a proposta e realizou a redação do artigo. Farias GS e Barros GR realizaram à escrita do artigo.

\section{Referências}

1. WorldHealth Organization.WHO.Global recommendations on physical activity for health. [Internet]. [citado em 2018 set 13]. Disponível em: http://www.who.int/dietphysicalactivity/ factsheet_recommendations/en/.

2. Sousa TF. Inatividade física em universitários brasileiros: uma revisão sistemática. Rev Atenção Saúde. 2011;9(29):47-55.

3. Santos SFS, Freitas Junior IF, Alvarenga AM, Fonseca SA, Virtuoso Junior JS, Sousa TF. Prevalence and factors associated with leisure-time physical activity: survey repeated in university students. Rev Bras Cineantropom Desempenho Hum. 2016;18(5):577-90.

4. Haase A, Steptoe A, Sallis JF, Wardle J. Leisure-time physical activity in university students from 23 countries: associations with health beliefs, risk awareness, and national economic development. Prev Med. 2004;39(1):182-90.

5. Sousa TF. Atividades físicas praticadas no lazer por universitários de uma instituição pública do nordeste do Brasil. Rev Bras Ativ Fis Saúde. 2012;17(2):125-31.

6. Cruz MS, Bernal RTI, Claro RM. Tendência da prática de atividade física no lazer entre adultos no Brasil (2006-2016). Cad Saúde Pública. 2018;34(10):e00114817.
7. Morseth B, Jacobsen BJ, Emaus N, Wilsgaard T, Jørgensen L. Secular trends and correlates of physical activity: The Tromsø Study 1979-2008. BMC Public Health. 2016;16(1):1215.

8. Instituto Nacional de Estudos e Pesquisas Educacionais Anísio Teixeira. INEP. Censo da Educação superior. 2017. [Internet]. [citado em 2019 ago 17]. Disponível em: http:// portal.inep.gov.br/web/guest/censo-da-educacao-superior.

9. Azevedo MR, Araújo CL, Silva MC, Hallal PC. Tracking of physical activity from adolescence to adulthood: a populationbased study. Rev Saúde Pública. 2007;41(1): 69-75.

10. Oliveira CS, Gordia AP, Quadros TMB, Campos W. Atividade física de universitários brasileiros: uma revisão da literatura. Rev Atenção Saúde. 2014;12(42):71-7.

11. Sousa TF, Santos SFS, José HPM. Barreiras percebidas à prática de atividade física no nordeste do Brasil. Pens Prát. 2010;13(1):1-15.

12. Franca C, Colares V. Estudo comparativo de condutas de saúde entre universitários no início e no final do curso. Rev Saúde Pública. 2008;42(3):420-7.

13. Sousa TF, Fonseca SA, José HPM, Nahas MV. Estudo MONISA: características e aspectos metodológicos. Rev Bras Epidemiol. 2012;15(4):904-7.

14. Sousa TF, Fonseca SA, José HPM, Nahas MV. Validade e reprodutibilidade do questionário Indicadores de Saúde e Qualidade de Vida de Acadêmicos (Isaq-A). Arq Cien Esp. 2013;1(1):21-30.

15. Brasil. Ministério da Educação. Coordenação de Aperfeiçoamento de Pessoal de Nível Superior. CAPES. Tabela de áreas de conhecimento/avaliação. Brasília: Ministério da Educação. 2018. [citado em 2019 ago 25]. Disponível em: https://www.capes.gov.br/avaliacao/ instrumentos-de-apoio/tabela-de-areas-do-conhecimentoavaliacao.

16. Franco DC, Sousa TF. Atividades físicas praticadas no lazer por universitários: prevalências e fatores associados. Arq Ciên Esp. 2018;6(2):78-81.

17. Malta DC, Moura EC, Castro AM, Cruz DKA, Neto OLM, Monteiro CA. Padrão de atividade física em adultos brasileiros: resultados de um inquérito por entrevistas telefônicas, 2006. Epidemiol Serv Saúde. 2009;18(1):7-16.

18. Carvalho CMM, Martelli A, Marchetti PH, Silva JJ, Magalhães RA. Efeito do treinamento de força em mulheres menopausadas portadoras de osteoporose: uma breve revisão. Rev Saúde em Foco. 2017; 4(2):40-56.

19. Sousa TF, Santos SFS, Rodrigues AS, Papini CB, Silva DAS, Tribess S. Prevalence and factors associated with body image dissatisfaction among university students: repeated surveys analysis. Rev Bras Cineantropom Desempenho Hum. 2019;21:e53036.

20. Rodrigues JVMS, Araújo FLM, Columá JF, Triani FS. JiuJitsu brasileiro: notas sobre a transposição da arte marcial para o esporte espetáculo. Arq Ciên Esp. 2018;6(1):11-4.

21. Alleoni BN. A manifestação corporal capoeira: a manifestação corporal capoeira: capoeira: uma cultura nacional brasileira. Rev Mackenzie Educ Fís Esporte. 2010; 9(1):24-31.

22. Netto JSC, Aptekmann NP. Efeitos do treinamento funcional sobre a composição corporal: um estudo em alunos fisicamente ativos de academia. Rev Bras Fisiologia do Exercício. 2016;15(2):68-76.

23. Dumith SC, Domingues MR, Gigante DP. Epidemiologia das atividades físicas praticadas no tempo de lazer por adultos do Sul do Brasil. Rev Bras Epidemiol. 2009;12(4):646-58. 
24. Bichescu A. Leisure time sport activities of the students at Resita University. Procedia Soc Behav Sci. 2014;117(9):735-40.

25. Oliveira CS, Stefane CA, Liziero L, Gabilan JG, Paranhos Filho AC, Guimarães FKO. A prática de atividade física na cidade universitária da Universidade Federal de Mato Grosso do Sul (UFMS). Ciênc Saúde Colet. 2011;16:1489-96.

26. Mezzaroba C, Pires GL. Breve panorama histórico do voleibol: do seu surgimento à espetacularização esportiva. Ativ Fís, Lazer Qual Vida: Rev Educ Fís. 2011;2(2):3-19.

27. Bergier J, Bergier B, Tsos A. Variations in physical activity of male and female students from the Ukraine in health-promoting life style. Ann Agric Environ Med. 2017;24(2):217-21.

28. Del Duca GF, Nahas MV, Hallal PC, Peres KG. Atividades físicas no lazer entre adultos de Florianópolis, Santa Catarina, Brasil: estudo populacional sobre as características das práticas e de seus praticantes. Ciênc Saúde Colet. 2014;19(11): 4595-604.
29. Teixeira IP,Nakamura PM, Kokubun E. Prática de caminhada no lazer e no deslocamento e associação com fatores socioeconômicos e ambiente percebido em adultos. Rev Bras Cineantropom Desempenho Hum. 2014;16(3):345-58.

30. Stattin K, Michaelsson K, Larsson SC, Wolk A, Byberg L. Leisure-time physical activity and risk of fracture: a cohort study of 66,940 men and women. J Bone Miner Res. 2017;32(8):1599-606.

Recebido: 07/01/2020

Aprovado: 01/05/2020

Como citar este artigo:

Sousa TF, Franco DC, Farias GS, Barros GR. Atividades físicas de lazer por universitários: inquéritos repetidos nos anos de 2010, 2012 e 2014. Rev Bras Ativ Fis Saúde. 2019;24:e0099. DOI: 10.12820/rbafs.24e0099 